Research Paper

\title{
Diagnostic Value of Plasma MicroRNAs for Lung Cancer Using Support Vector Machine Model
}

\author{
Wei Wang1,2, Mingcui Ding1, Xiaoran Duan³, Xiaolei Feng'1, Pengpeng Wang1, Qingfeng Jiang4, Zhe \\ Cheng, ${ }^{5}$, Wenjuan Zhang, ${ }^{6}$, Songcheng $\mathrm{Yu}^{7}$, Wu Yao ${ }^{1}$, Liuxin $\mathrm{Cui}^{3}$, Yongjun $\mathrm{Wu}^{7}$, Feifei Feng ${ }^{8}$, Yongli \\ Yang ${ }^{9 凶}$ \\ 1. Department of Occupational Health and Occupational Disease, College of Public Health, Zhengzhou University, Zhengzhou, China \\ 2. The Key Laboratory of Nanomedicine and Health Inspection of Zhengzhou, Zhengzhou, China \\ 3. Department of Environmental Health, College of Public Health, Zhengzhou University, Zhengzhou, China \\ 4. Department of Thoracic Surgery, the Affiliated Cancer Hospital of Zhengzhou University (Henan Cancer Hospital), Zhengzhou, China \\ 5. Department of Respiratory Medicine, The First Affiliated Hospital of Zhengzhou University, Zhengzhou, China \\ 6. Department of Public Health and Preventive Medicine, School of Medicine, Jinan University, Guangzhou, China \\ 7. Department of Sanitary Chemistry, College of Public Health, Zhengzhou University, Zhengzhou, China \\ 8. Department of Health Toxicology, College of Public Health, Zhengzhou University, Zhengzhou, China \\ 9. Department of Epidemiology and Biostatistics, College of Public Health, Zhengzhou University, Zhengzhou, China \\ $\square$ Corresponding author: Yongli Yang, PhD. Department of Epidemiology and Biostatistics, College of Public Health, Zhengzhou University, Zhengzhou, \\ China. Tel: +86 371 67781466; Fax: +86 371 67781868; E-mail: ylyang377@126.com
}

(C) The author(s). This is an open access article distributed under the terms of the Creative Commons Attribution License (https://creativecommons.org/licenses/by/4.0/). See http://ivyspring.com/terms for full terms and conditions.

Received: 2018.10.09; Accepted: 2019.06.25; Published: 2019.08.28

\begin{abstract}
Aim: Small single-stranded non-coding RNAs (miRNAs) play an important role in carcinogenesis through degrading target mRNAs. However, the diagnostic value of miRNAs was not explored in lung cancers. In this study, a support-vector-machine (SVM) model for diagnosis of lung cancer was established based on plasma miRNAs biomarkers, clinical symptoms and epidemiology material.

Methods: The expressions of plasma miRNA were examined with SYBR Green-based quantitative real-time PCR.

Results: We identified that the expressions of 10 plasma miRNAs (miR-21, miR-20a, miR-210, miR-145, miR-126, miR-223, miR-197, miR-30a, miR-30d, miR-25), smoking status, fever, cough, chest pain or tightness, bloody phlegm, haemoptysis, were significantly different between lung cancer and control groups $(P<0.05)$. The accuracies of the combined SVM, miRNAs SVM, symptom SVM, combined Fisher, miRNAs Fisher and symptom Fisher were $96.34 \%, 80.49 \%, 84.15 \%, 84.15 \%, 75.61 \%$, and $80.49 \%$, respectively; AUC of these six model were $0.976,0.841,0.838,0.865,0.750$, and 0.801 , respectively. The accuracy and AUC of combined SVM were higher than the other 5 models $(P<0.05)$.

Conclusions: Our findings indicate that SVM model based on plasma miRNAs biomarkers may serve as a novel, accurate, noninvasive method for auxiliary diagnosis of lung cancer.
\end{abstract}

Key words: Lung cancer, Plasma miRNAs, Support vector machine, Diagnosis

\section{Introduction}

Lung cancer is currently the number one cause of morbidity and mortality worldwide [1], which has been classified into small cell lung cancer (SCLC) and non-small-cell lung cancer (NSCLC). NSCLC could account for $85 \%$ of all lung cancers to become the main subgroup of lung cancer [2]. NSCLCs can be further divided into adenocarcinoma (AC), squamous cell carcinoma (SCC) and large cell carcinoma (LCLC) three major histological subtypes [3]. Yet the NSCLC patients at an early stage with no obvious clinical symptoms, and lack of sensitive biomarkers and effective tools for early diagnosis, therefore more than $75 \%$ of NSCLC patients are still diagnosed at advanced stages with distant metastases ${ }^{[4]}$. Although novel therapies are improving the survival of lung cancer patients, 5-year survival rate was still less than 
$15 \%$ for advanced NSCLCs. However, the 5-year survival rate was up to $80 \%$ for the initial stage NSCLCs [5]. Therefore, early diagnosis and early screening of lung cancer are particularly important.

Lung cancer is diagnosed by means of histological examination, diagnostic imaging, low-dose spiral computed tomography (LDSCT) and positron emission tomography (PET). Although these techniques have been improved, they still have some limitations and the five-year death rate of lung cancer remains low ${ }^{[6]}$. For example, histological examination is the golden standard for diagnosis of lung cancer, but it is not suitable for early screening of lung cancer because of its traumatic and highly technical requirements. Diagnostic imaging such as Chest $X$ Ray (CxR) and Computed Tomography (CT) have been used for diagnosing NSCLC at an early stage, however, there is a certain radiation hazard and limited role in reducing lung cancer mortality [7]. While lung cancer mortality is reduced by $20 \%$ in high-risk lung cancer patients through LDSCT method, the false-positive are as high as 90\% [8]. Although the sensitivity and specificity of PET method are up to $90 \%$, there is still a $10 \%$ false-positive rate and the cost is expensive [9]. Therefore, new biomarkers and therapeutic strategies urgently need to be developed for better management of lung cancer.

miRNAs are small single-stranded non-coding RNAs that play vital regulatory roles by targeting mRNAs for degradation or translational repression. It acts as key regulators of cell proliferation, differentiation, apoptosis and other biological processes ${ }^{[10]}$. A line of studies suggest that miRNAs are involved in human diseases and cancers. miRNAs expression is associated with lung cancer has been identified in varieties of normal and cancer tissues [11, 12]. Moreover, it has been demonstrated that plasma miRNAs regulate numerous target genes and play a critical role in lung carcinogenesis, which indicates that miRNAs might be a potential diagnostic tool for lung cancer [13]. Published studies [14-16] have shown that 11 plasma miRNAs (miR-16, miR-21, miR-20a, miR-210, miR-145, miR-126, miR-223, miR-197, miR-30a, miR-30d, miR-25) in lung cancer patients are abnormal expressions. These results suggest that combined with several miRNAs can improve the sensitivity and specificity for the early diagnosis of lung cancer.

Data mining(DM), also called Knowledge Discovery in Database(KDD), is extracting potentially useful information and knowledge of the process from abundant, incomplete, noisy, fuzzy and random practical application data [17]. DM has a unique advantage in solving multi-parameter problems.
Classification is part of the important functions of data mining, which is often closely related to disease diagnosis. At present, data mining is used primarily in the field of auxiliary diagnosis of diseases [18]. DM techniques include SVM, artificial neural networks (ANN), decision tree (DT), genetic algorithms and so on. SVM is a pattern recognition method based on statistical learning theory (SLT) and structural risk minimization, which has several advantages such as prominent generalization ability and non-linear processing capacity and high-dimensional processing capacity in many areas [19].

Based on the previous research [20], this study explored the significance of the SVM model by using data of plasma miRNAs biomarkers for the auxiliary diagnosis of lung cancer.

\section{Materials and methods}

\section{Study population}

The lung cancer patient group consisted of 148 cases (age rank 29-87 years) with primary lung cancer from the First Affiliated Hospital of Zhengzhou University, Henan Cancer Hospital and Henan Provincial Chest Hospital, from Jun. 2016 to Feb. 2017. Patients were selected on the basis of the following inclusion criteria: (1) patients had a pathological diagnostic primary lung cancer that met histological or cytological criteria; (2) without undergoing surgical resection, chemotherapy, or radiotherapy; (3) without previous other organ tumors; (4) good compliance and availability of outcome data. Patients were excluded with major organ function failure, pregnant, or lactating. Pathologic diagnosis was based on WHO criteria. Lung cancer staging for each patient was performed according to the AJCC Cancer Staging Manual, 7th edition.

Controls come from a company who take physical examinations in Qixian Center for Disease Control and Prevention. The controls were excluded according to the following criteria: (1) without malignant tumors of the lung or other organs; (2) without major organ function failure; (3) without pregnant or lactating; (4) good compliance and availability of outcome data. A total of 148 genderand age-frequency matched ( \pm 3 years) were enrolled in this study. The permission was got from each participant. A questionnaire that included the information of epidemiology was completed for each participant by trained interviewers. Smokers are defined as people who have smoked for six months or more in their lifetime according to the criteria of WHO. The alcohol-drinkers are defined as drinking alcohol at least once a week and the consumption of pure alcohol is above $20 \mathrm{~g}$. 


\section{Main instruments and reagents}

The instruments and reagents used in the study included a Labcycler PCR amplifier (SensoQuest Company, China), a 7500 Fast Real-time PCR system (ABI, America), primers (Sangon Biotech), miRcute miRNA extraction and separation kit(Tiangen, Beijing), MiRcute enhanced miRNA fluorescence quantitative detection kit(Tiangen, Beijing) and ChemiDoc MP gel imaging analyzer(Bio-RAD, America).

\section{Statistical analysis and model evaluation}

The $\mathrm{Ct}$ values of the samples were calculated with the software for real-time PCR instrument. The comparison of multiple of the expression of miRNA in the lung cancer patients to the normal controls was calculated using the formula of $2^{-\Delta \Delta \mathrm{Ct}}\left(\Delta \mathrm{Ct}=\mathrm{Ct}_{\mathrm{miR}}-\right.$ $C t_{\text {external reference; }} \Delta \Delta \mathrm{Ct}=\Delta \mathrm{Ct}_{\mathrm{miR}}-\Delta \mathrm{Ct}_{\text {average normal controls }}$ ).

The data was analyzed using SPSS 21.0 software. SPSS Clementine 21.0 software was used for data mining. The analysis of the quantitative data was analyzed with independent sample t-test or Mann-Whitney U. Each contingency table was tested by Chi-Square test. Binary logistic regression was conducted to analyse the influencing factors of lung cancer. The significance level was set at 0.05 .

This study assessed sensitivity, specificity, accuracy positive predictive value (PPV), negative predictive value (NPV), and area under the ROC curve (AUC) to estimate the models.

\section{Establishment of models}

\section{Data preprocessing}

Data transformation: The relative expression of 11 miRNAs did not follow a normal distribution, so normal transformation was needed. The expression of 11 miRNAs was normalized based on 10 common logarithm transformations.

Groups of training set and validation set: Based on the random sampling function of the partition node, according to a ratio of 3:1, the normalized data of each group were separated randomly into a training set (114 controls, 100 cancer cases) and a validation set (34 controls, 48 cancer cases). The training set was utilized to develop the model, while the validation set was used to verify the model.

\section{Model derivation}

The Data node is the source of data for the study; the variables are documented using Type node; the samples were randomly divided into the training set and validation set according to the proportion of 3:1 using Partition node; Random number seed is 1111111.
The Fisher and SVM models were developed using the training set, then the samples of the validation set were used to validate the quality of the models. The Combined model (16 items) with 16 input variables of smoking status, fever, cough, chest pain or tightness, bloody phlegm, hemoptysis and expressions of 10 plasma miRNAs (miR-21, miR-20a, miR-210, miR-145, miR-126, miR-223, miR-197, miR-30a, miR-30d, miR-25); the miRNAs model (10 items) with 10 input variables of plasma miRNAs (miR-21, miR-20a, miR-210, miR-145, miR-126, miR-223, miR-197, miR-30a, miR-30d, miR-25); the symptom model (6 items) with 6 input variables of fever, cough, chest pain or tightness, bloody phlegm, hemoptysis.

\section{Fisher discrimination model}

Fisher discrimination is a widely used classification model in traditional statistical methods. The basic idea: Projection before discriminant analysis, Projection is the core of the Fisher discrimination analysis. After repeating training, the Fisher discrimination parameter settings were: Use partitioned data: no; method: Enter; Mode: Expert; Prior probabilities: All groups equal; Use covariance matrix: Within-groups.

\section{SVM model}

The basic principle is to transform the input space into a high dimensional space by using the nonlinear transformation defined by the inner product function, and to find the optimal linear classification surface.

After repeating training, the SVM parameter settings were: Use partitioned data: no; Mode: Expert; Kernel type: Polynomial; Gamma: 1; Stopping criteria: $1.0 \mathrm{E}-3$.

\section{Results}

\section{Demographic characteristics of lung cancer patients and controls}

The 148 lung cancer patients (mean age $60.97 \pm$ 10.83 years) and 148 controls (mean age $60.14 \pm 9.66$ years) were enrolled. The age distribution of subjects was in normal distribution, so the age group was divided into two groups according to mean age (60 years). All the subjects were divided into four groups (Never smoking; Light smoking: <10 cigarettes/day; Moderate smoking: 10 20 cigarettes/day; Heavy smoke $>20$ cigarettes/day) according to the smoking status. As shown in Table 1, the average age, sex and alcohol were no significant differences between the two groups $(P>0.05)$. However, the frequency of smoking, fever, cough, chest pain or tightness, bloody 
phlegm and hemoptysis were significantly higher in the cancer group than that in control group $(P<0.001)$.

Table 1. Demographic characteristics of lung cancer patients and controls

\begin{tabular}{|c|c|c|c|c|c|}
\hline Variable & & $\begin{array}{l}\text { Lung cancer } \\
(\mathrm{n}=148)\end{array}$ & $\begin{array}{l}\text { Controls } \\
(\mathrm{n}=148)\end{array}$ & $x^{2} / t$ & $P$ \\
\hline Age $^{*}$ & & $60.97 \pm 10.83$ & $60.14 \pm 9.66$ & 0.691 & 0.490 \\
\hline \multirow[t]{2}{*}{ Age-grouped } & $\leq 60$ & 67 & 68 & 0.014 & 0.907 \\
\hline & $>60$ & 81 & 80 & & \\
\hline \multirow[t]{2}{*}{ Gender } & Male & 98 & 99 & 0.821 & 0.365 \\
\hline & Female & 50 & 49 & & \\
\hline \multirow[t]{2}{*}{ Fever } & No & 131 & 146 & 12.654 & $<0.001$ \\
\hline & Yes & 17 & 2 & & \\
\hline \multirow[t]{2}{*}{ Cough } & No & 57 & 130 & 77.387 & $<0.001$ \\
\hline & Yes & 91 & 18 & & \\
\hline \multirow{2}{*}{$\begin{array}{l}\text { Chest pain or } \\
\text { tightness }\end{array}$} & No & 86 & 135 & 42.878 & $<0.001$ \\
\hline & Yes & 62 & 13 & & \\
\hline \multirow[t]{2}{*}{ Bloody phlegm } & No & 109 & 148 & 44.918 & $<0.001$ \\
\hline & Yes & 39 & 0 & & \\
\hline \multirow[t]{2}{*}{ Hemoptysis } & No & 134 & 148 & 14.695 & $<0.001$ \\
\hline & Yes & 14 & 0 & & \\
\hline \multirow[t]{2}{*}{ Weak } & No & 146 & 145 & 0.000 & 1.000 \\
\hline & Yes & 2 & 3 & & \\
\hline \multirow[t]{2}{*}{ Alcohol } & Never & 134 & 129 & 0.853 & 0.356 \\
\hline & Yes & 14 & 19 & & \\
\hline \multirow[t]{4}{*}{ Smoking status } & Never & 76 & 89 & 16.989 & $<0.001$ \\
\hline & Light & 10 & 26 & & \\
\hline & Moderate & 28 & 15 & & \\
\hline & Heavy & 34 & 18 & & \\
\hline
\end{tabular}

Note: The * indicates age according with normal distribution.

\section{Clinical pathologic characteristics of lung cancer patients}

The clinical and pathological characteristics of lung cancer patients collected in this study are shown in Table 2. The lung cancer group was consisted of 36 SCC cases, 18 SCLC cases, 66 AC cases, 2 LCLC cases, and 26 other histological type cases; 33 cases of clinical stage I and II, 101 cases of clinical stage III and IV.

\section{Comparison of the expressions of 11 plasma miRNAs between the two groups}

As seen in Table 3, expressions of 10 plasma miRNAs (miR-21, miR-20a, miR-210, miR-145, miR-126, miR-223, miR-197, miR-30a, miR-30d, miR-25) were all significantly up-regulated in lung cancer patients than controls $(P<0.05)$. However, the expression of miR-16 was no significant difference between lung cancer patients and controls $(P>0.05)$.

\section{Data mining}

The data of the Fisher and SVM model based on the smoking status, fever, cough, chest pain or tightness, bloody phlegm, haemoptysis and expressions of 10 plasma miRNAs (miR-21, miR-20a, miR-210, miR-145, miR-126, miR-223, miR-197, miR-30a, miR-30d, and miR-25) are presented in Table 4. In the training set, the accuracies of combined Fisher, miRNAs Fisher, symptom Fisher, combined SVM, miRNAs SVM, and symptom SVM model were
$87.38 \%, 74.77 \%, 79.91 \%, 98.13 \%, 85.51 \%$, and $83.64 \%$, respectively. The accuracies in the validation set were $84.15 \%, 75.61 \%, 80.49 \%, 96.34 \%, 80.49 \%$, and $84.15 \%$, respectively.

Table 2. Clinical and pathological characteristics of lung cancer patients and controls

\begin{tabular}{llll}
\hline \multicolumn{2}{l}{ Clinical and pathological characteristics } & $\mathrm{n}$ & Percentage (\%) \\
\hline Histological type & SCLC & 18 & 12.16 \\
& SCC & 36 & 24.32 \\
& AC & 66 & 44.59 \\
& LCLC & 2 & 11.35 \\
& Others & 26 & 17.57 \\
TNM stage* & I+II & 33 & 24.63 \\
& III+IV & 101 & 75.37 \\
lymphatic & No & 21 & 17.80 \\
metastasis $^{*}$ & Yes & 97 & 82.20 \\
distant metastases $^{*}$ & No & 83 & 70.34 \\
& Yes & 35 & 29.67
\end{tabular}

Note: The * indicates data is missing.

Table 3. The relative expression of 11 plasma miRNAs in lung cancer and controls

\begin{tabular}{|c|c|c|c|c|}
\hline miRNAs & $\begin{array}{l}\text { Lung cancer }(n=148) \\
\mathrm{M}(P 25, P 75)\end{array}$ & $\begin{array}{l}\text { Control }(n=148) \\
\mathrm{M}(P 25, P 75)\end{array}$ & Z & $P$ \\
\hline miR-16 & $1.60(0.70,2.93)$ & $1.39(0.66,2.51)$ & -1.184 & 0.236 \\
\hline miR-21 & $1.05(0.77,2.09)$ & $0.68(0.53,0.90)$ & -6.017 & $<0.001$ \\
\hline miR-20a & $1.93(0.81,4.40)$ & $0.80(0.42,1.51)$ & -6.264 & $<0.001$ \\
\hline miR-210 & $1.10(0.53,3.09)$ & $0.68(0.39,1.24)$ & -4.267 & $<0.001$ \\
\hline miR-145 & $1.11(0.56,2.93)$ & $0.70(0.44,1.07)$ & -4.242 & $<0.001$ \\
\hline miR-126 & $1.64(0.71,2.83)$ & $0.77(0.32,1.58)$ & -5.096 & $<0.001$ \\
\hline miR-223 & $2.26(1.26,5.55)$ & $0.76(0.41,1.36)$ & -8.952 & $<0.001$ \\
\hline miR-197 & $1.13(0.59,2.29)$ & $0.59(0.41,1.25)$ & -5.008 & $<0.001$ \\
\hline miR-30a & $0.82(0.51,2.81)$ & $0.66(0.37,1.75)$ & -2.908 & $<0.001$ \\
\hline miR-30d & $1.37(0.78,3.55)$ & $0.69(0.48,1.24)$ & -6.409 & $<0.001$ \\
\hline miR-25 & $1.36(0.77,3.27)$ & $0.80(0.34,1.73)$ & -4.925 & $<0.001$ \\
\hline
\end{tabular}

Table 4. Effect of data mining on distinguish lung cancer

\begin{tabular}{|c|c|c|c|c|c|}
\hline \multirow[t]{2}{*}{ Model } & & \multicolumn{2}{|c|}{ Training set $(\mathrm{n}=214)$} & \multicolumn{2}{|c|}{ Validation set $(\mathrm{n}=82)$} \\
\hline & & $\begin{array}{l}\text { Cancer } \\
\text { cases }\end{array}$ & Controls & $\begin{array}{l}\text { Cancer } \\
\text { cases }\end{array}$ & Controls \\
\hline \multirow{4}{*}{$\begin{array}{l}\text { Combined Fisher } \\
\text { model }\end{array}$} & Cancer cases & 79 & 21 & 41 & 7 \\
\hline & Controls & 6 & 108 & 6 & 28 \\
\hline & Total & 85 & 129 & 47 & 35 \\
\hline & Accuracy & $87.38 \%$ & & $84.15 \%$ & \\
\hline \multirow[t]{4}{*}{ miRNAs Fisher model } & Cancer cases & 70 & 30 & 38 & 10 \\
\hline & Controls & 24 & 90 & 10 & 24 \\
\hline & Total & 94 & 120 & 48 & 34 \\
\hline & Accuracy & $74.77 \%$ & & $75.61 \%$ & \\
\hline \multirow[t]{4}{*}{ Symptom Fisher model } & Cancer cases & 71 & 29 & 38 & 10 \\
\hline & Controls & 14 & 100 & 6 & 28 \\
\hline & Total & 85 & 129 & 44 & 38 \\
\hline & Accuracy & $79.91 \%$ & & $80.49 \%$ & \\
\hline \multirow[t]{4}{*}{ Combined SVM model } & Cancer cases & 99 & 1 & 47 & 1 \\
\hline & Controls & 3 & 111 & 2 & 32 \\
\hline & Total & 102 & 112 & 49 & 33 \\
\hline & Accuracy & $98.13 \%$ & & $96.34 \%$ & \\
\hline \multirow[t]{4}{*}{ miRNAs SVM model } & Cancer cases & 83 & 17 & 38 & 10 \\
\hline & Controls & 14 & 100 & 6 & 28 \\
\hline & Total & 97 & 117 & 44 & 38 \\
\hline & Accuracy & $85.51 \%$ & & $80.49 \%$ & \\
\hline \multirow[t]{4}{*}{ Symptom SVM model } & Cancer cases & 81 & 19 & 42 & 6 \\
\hline & Controls & 16 & 98 & 7 & 27 \\
\hline & Total & 97 & 117 & 49 & 33 \\
\hline & Accuracy & $83.64 \%$ & & $84.15 \%$ & \\
\hline
\end{tabular}




\section{The evaluation of models}

The results of the evaluation indexes of the 6 models were presented in Table 5. Sensitivity of combined SVM model reached $97.90 \%$, and the specificity was $94.10 \%$. PPV and NPV were likewise highest. Meanwhile, AUC was greater than 0.9. On the other hand, AUC of the miRNAs Fisher and symptom Fisher models were slightly smaller than the other models.

Table 5. Comparison results in the validation set by SVM and Fisher models

\begin{tabular}{|c|c|c|c|c|c|c|c|}
\hline Model & & $\begin{array}{l}\text { Sensitivity } \\
(\%)\end{array}$ & $\begin{array}{l}\text { Specificity } \\
(\%)\end{array}$ & $\begin{array}{l}\text { Accuracy } \\
(\%)\end{array}$ & $\begin{array}{l}\text { PPV } \\
(\%)\end{array}$ & $\begin{array}{l}\text { NPV } \\
(\%)\end{array}$ & AUC $(95 \%$ CI $)$ \\
\hline \multirow[t]{3}{*}{ Fisher } & Combined & 0.854 & 0.824 & 0.842 & 0.872 & 0.800 & $0.865(0.821,0.902)$ \\
\hline & miRNAs & 0.792 & 0.706 & 0.756 & 0.792 & 0.706 & $0.750(0.697,0.798)$ \\
\hline & Symptom & 0.792 & 0.824 & 0.805 & 0.864 & 0.737 & $0.801(0.751,0.845)$ \\
\hline \multirow[t]{3}{*}{ SVM } & Combined & 0.979 & 0.941 & 0.963 & 0.959 & 0.970 & $0.976(0.952,0.990)$ \\
\hline & miRNAs & 0.792 & 0.824 & 0.805 & 0.864 & 0.737 & $0.841(0.795,0.881)$ \\
\hline & Symptom & 0.875 & 0.794 & 0.842 & 0.857 & 0.818 & $0.838(0.791,0.878)$ \\
\hline
\end{tabular}

The results of the AUC of the 6 models were shown in Table 6 . The AUC of combined SVM model was superior to the other 5 models, and the difference was statistically significant $(P<0.05)$; The AUC of combined Fisher model was higher than miRNAs Fisher model and symptom Fisher model $(P<0.05)$. There were no statistical differences in AUC among the other 3 models $(P>0.05)$.

Table 6. Comparison of results in validation set by SVM and Fisher discriminant analysis

\begin{tabular}{lll}
\hline Comparison of models & $\mathrm{Z}$ & $P$ \\
\hline Combined SVM model vs Combined Fisher model & 5.474 & $<0.0001$ \\
Combined SVM model vs miRNAs SVM model & 6.445 & $<0.0001$ \\
Combined SVM model vs Symptom SVM model & 6.363 & $<0.0001$ \\
miRNAs SVM model vs Symptom SVM model & 0.105 & 0.9168 \\
miRNAs SVM model $v$ s miRNAs Fisher model & 4.032 & 0.0001 \\
Symptom SVM model $v$ s Symptom Fisher model & 2.256 & 0.0241 \\
Combined Fisher model $v s$ miRNAs Fisher model & 4.179 & $<0.0001$ \\
Combined Fisher model $v$ s Symptom Fisher model & 3.167 & 0.0015 \\
miRNAs Fisher model vs Symptom Fisher model & 1.454 & 0.1459 \\
\hline
\end{tabular}

\section{Discussion}

Early diagnosis and effective treatment of lung cancer is the key to improve the survival rate of patients. Therefore, early and non-invasive biomarkers for lung cancer diagnosis have been the most popular research areas. It has been shown that circulating miRNAs are stable under the actual experimental conditions and that abnormal expression of cancer-related miRNAs may be earlier than the clinical symptoms, therefore, circulating miRNAs may be used as tumor biomarkers [21]. A large body of studies have suggested that a series of circulating miRNAs have the potential as diagnostic tool in malignancies ${ }^{[22]}$. It has been shown that four plasma miRNAs (miR-21, miR-126, miR-210, and miR-486) could differentiate NSCLC from controls with $86.22 \%$ sensitivity and $96.55 \%$ specificity, which also could to distinguish NSCLC with $73.33 \%$ sensitivity and $96.55 \%$ specificity in phase I [23]. In the plasma of NSCLC patients, one study identified 15 types of miRNAs associated with lung cancer tissues from the literature and found that the expression of miR-155, miR-197, and miR-182 were significant increase in phase I [16]. The sensitivity and specificity of diagnosis NSCLC patients were $81.33 \%$ and $86.76 \%$, respectively.

In this study, we compared the expression of 11 plasma miRNAs in lung cancer patient to that in the controls. Single-factor analysis showed that the expressions of 10 plasma miRNAs (miR-21, miR-20a, miR-210, miR-145, miR-126, miR-223, miR-197, miR-30a, miR-30d, miR-25) in lung cancer group were statistically significant higher than the controls; Multiple factor analysis revealed that elevated plasma miR-20a levels and miR-223 were risk factors for lung cancer.

The ten miRNAs explored in the present study have been evaluated as lung cancer markers in other studies. Potential mechanisms of the miRNAs on lung cancer were explored in previous study (Seen in Table 7), the main signaling pathways including PI3K/Akt/NF-Kb (miR-21, miR-223, miR-145, miR-126, and miR-30a) [24-28], STAT3 (miR-126, and miR-197) [27, 29, 30], estrogen (miR-21 and miR-210) [31], et al.

Table 7. Potential mechanisms of the miRNAs on lung cancer

miRNAs Pathways

miR-20a angiogenesis, TGF- $\beta$ pathway, platelet-derived growth factor pathway, and oxidative stress response ${ }^{[32]}$

miR-21 PI3K/AKT/NF-KB signaling pathway, and estrogen signaling pathway [24]; autophagy-related AMPK/ULK1 signaling pathway[56]

miR-210 estrogen signaling pathway ${ }^{[31]}$

miR-223 Notch/miR-223/FBXW7 pathway[5]; NF-kB signaling pathway[58]; IGF-1R/Akt/S6 signaling pathwayl25]; IGF-1R/PI3K/AKT signaling pathway ${ }^{[59]}$

miR-25 ERK signaling pathway[60]; cell cycle regulation[61]

miR-145 ERß/MALAT1/miR145-5p/NEDD9 signaling pathway[62]; EGFR/PI3K/AKT signaling pathway[22]; JNK signaling pathway [36]; mTOR signaling pathwayl[64]

miR-126 STAT3 signal pathway ${ }^{[30] ;}$ PI3K/AKT/Snail signal pathway ${ }^{[27]}$ miR-30a PI3K/AKT signaling pathway[28]

miR-197 miR-197/CKS1B/STAT3-mediated PD-L1 network [29]

Transcription factors such as HMBOX1, DDX5, and ZBTB5, which were identified to be co-regulated by miR-20a and miR-15b, have implication on cancer progression [32]. E2F1 and E2F8 belong to E2F transcription factor family that is essential for the regulation of cell cycle progression [33]. miR-20a has been shown to directly inhibit E2F1 transcription factor and highly express in NSCLC tissues [34]. miR-223 suppressed the proliferation, migration and 
invasion of NSCLC cells through directly inhibition of E2F8 expression [35]. Increased expression of miR-20a and miR-223 were found in lung cancer in our study, which could be used as a molecular biomarker in auxiliary diagnosis of lung cancer.

miR-25, acting as an oncogene or anti-oncogene located on chromosome $7 \mathrm{p} 22.1$, is involved in the development of multiple malignant tumors at the post-transcriptional level ${ }^{[36]}$. The expression of miR-25 in NSCLC tissues was significantly higher than that in adjacent non-cancerous tissues, which plays a carcinogenic effect by regulating cell cycle element E2 and is associated with cancer cell for resistance, proliferation, metastasis, invasion, and so on [37]. miR-21 is over expressed in a variety of diseases and negatively responsible for the regulation of tumor suppressor genes by participating in the proliferation, invasion, metastasis and vascular infiltration of tumor cells, thus promoting the development of tumor [38]. miR-210 and hypoxia-inducible factor 1-alpha (HIF-1a) play a synergistic role in the proliferation, differentiation, apoptosis, angiogenesis, DNA damage repair and energy metabolism of the hypoxic cell [39]. Study also demonstrated the high expression of miR-210 in advanced lung cancer [40]. miR-197 is related to infiltration and metastasis of tumor cells and located on chromosome 1p13.3. Recent research bears out the miR-197/CKS1B/STAT3-mediated PD-L1 network in chemoresistant NSCLC, independent of immunosuppression signals [29]. Another research found decreased expression of miR-197 induces p53-dependent lung cancer cell apoptosis, which may be oncogene [41]. The results of the study are basically in line with these studies in which the increased expression of miR-25, miR-21, miR-197 and miR-210 in the plasma of lung cancer compared with controls. Taken together, these results reflect the reliability and stability of miR-25, miR-21, miR-197 and miR-210 as the lung cancer biomarkers.

The data of the expressions of miR-145 are not consistent in different studies. It has been shown that miR-145 is down-regulated in various malignancies including lung adenocarcinoma, which inhibited cell proliferation through targeting epidermal growth factor receptor (EGFR) and nucleoside diphosphate 1 (NUDT1) [42]. However, Study found that the increased expression of miR-145 in the plasma lung cancer, which is consistent with our study ${ }^{[43]}$. miR-126 may inhibit the proliferation of lung cancer cells and the expression of miR-126 was lower than normal tissue [44]. miR-30a can inhibit the invasion and migration of lung cancer cells by directly inhibiting the expression of the snail [45]. miR-30d could inhibit the cell proliferation and activity of NSCLC by directly regulating CCNE2 [46]. In this study, the relative expressions of miR-126, miR-30a and miR-30d in plasma lung cancer patients were greater than controls, and the data differed from the studies above.

Various data mining algorithms have been improved in recent years, such as cluster analysis, decision tree and rough set, ANN and genetic algorithm, SVM and fuzzy processing technology [47]. Each method has advantages and limits as well as the applicable scope. Fisher discriminant analysis is one of the most widely used method in multivariate statistical pattern recognition, which requires the independent input variables without interaction effect and normal distribution and so on [48]. Therefore, the analysis of the nonlinear system has a couple of limitations. In order to get the best generalization ability, based on the statistical learning theory of VC (Vapnik-Cher-Vonenkis) and structural risk minimization principle, SVM finds the best compromise between the complexity of the model and the ability to learn [49]. SVM is a classical method in data mining. There are several advantages of SVM method. For example, structural risk minimization and good generalization ability, what is based on statistical learning theory [50]. The second, SVM can achieve similar results with different kernel functions like ANN, which depends on the selected model [51]. In general, SVM is the optimal solution in the existing information situation, which makes up for the deficiency of ANN in determining the reasonable structure and local optimal problem, and has a significant improvement in learning methods. This study deeply analyzed with more mature SVM algorithms employed in the medical field.

At present, some studies have mostly focused on one or several biomarkers using traditional analysis methods. One study explored serum miR-22, miR-125b, and miR-15b diagnosis compared with the current commonly used tumor marker CEA, which indicates that the diagnostic significance of these three serum miRNAs(AUC=0.725, 0.704, and 0.619) for NSCLC was higher than that of serum CEA (AUC $=0.594)^{[52]}$. Meanwhile, some studies focused on gene and other biomarkers using ANN or decision tree model and so on. The ANN and decision tree model of lung cancer based on the genetic polymorphism of CYP1A1, GSTM1, $\mathrm{mEH}$, XRCC1, the length of telomere, and the methylations of p16 and RASSF1A gene, the results showed that the accuracy for ANN and decision tree model validation sets was $89.62 \%$ and $93.00 \%$ [53]. The accuracy and sensitivity were also improved by the above methods. In this study, the SVM model and Fisher model were established based on miRNAs tumor biomarkers and clinical symptom characteristics for the first time. 
We established the Fisher model with 10 miRNA and 6 symptom for lung cancer diagnostic, and, the AUCs of three models are combined Fisher model (16 items) $(0.865,95 \% \mathrm{CI}=0.821-0.902)$, miRNAs Fisher model (10 items) $(0.750,95 \% \mathrm{CI} 0.697-0.798)$, and symptom Fisher model (6 items) $(0.801,95 \% \mathrm{CI}$ $0.751-0.845)$, respectively. The accuracy for three model validation sets was $84.15 \%, 75.61 \%$, and $80.49 \%$, respectively. The combined Fisher model showed good ability to detect lung cancer, which is superior to the lung cancer diagnosis Fisher model (0.670, 95\%CI 0.569-0.761) established with FHIT, RASSF1A, p16 promoter methylation, and relative telomere length in our prophase research ${ }^{[20]}$. This may be due to the miRNAs biomarkers has better specificity compared with gene or other biomarkers. Our findings indicate that the changed expression levels could be used as potential biomarkers for diagnosis of lung cancer. Besides, probably because of the data pretreatment before model established. After the normal transformation, the expression levels of miRNAs are approximately normal distribution and without missing values.

miRNAs play a critical role in lung cancer carcinogenesis, which were studied widely as cancer biomarkers. Zhang et al [54] established screening method for early-stage NSCLC using four miRNAs (miR-145, miR-20a, miR-21, miR-223), and the AUC of the model was 0.897 . To the best of our knowledge, there is no data mining model for lung cancer diagnosis based on miRNAs. SVM model were established for lung cancer diagnostic in our study, which combined 10 miRNAs and 6 symptoms, had a higher accuracy. The combined SVM model with miRNAs was superior in lung cancer diagnosis in this study compared to models with methylation and telomere biomarkers in our prophase research ${ }^{[20]}$. The accuracy and AUC of combined SVM model in our study were also better than the results of other studies on gene and other biomarkers using ANN or SVM and so on. For example, one study explored eighteen genes (including TTN, RHOH, RPS20, TRBC2) for six cancer (including lung cancer) using SVM with accuracy of $75.10 \%$ [55].

As to the three models we established, the accuracy of models (10 miRNAs SVM, 6 symptom SVM model, and combined SVM) were $80.45 \%$, $84.15 \%$, and $96.34 \%$, respectively; the AUC of models (10 miRNAs SVM, 6 symptom SVM model, and combined SVM) were 0.841, 0.818, and 0.976, respectively. The AUC and accuracy of combined SVM model were better than the miRNAs SVM and symptom SVM model. Overall, the SVM model based on miRNAs and clinical symptom characteristics has a higher accuracy rate and might be useful for early diagnosis of lung cancer, which also has excellent predictive power, such as all patients with stage I and II lung cancer in validation set were correctly predicted to be lung cancer.

This study showed that 10 plasma miRNAs expression levels were associated with lung cancer, which provides a theoretical possibility for further prospective studies or large-scale clinical trials. More importantly, the expression of the plasma miRNAs is very stable under different harsh conditions, which indicating that the plasma miRNAs has the potential to serve as biomarker for auxiliary diagnosis of lung cancer. Our findings indicate that SVM model based on plasma miRNAs biomarkers may serve as a novel, accurate, noninvasive method for auxiliary diagnosis of lung cancer. However, there are some limitations in this study. Firstly, the selection of 10 plasma miRNAs were based on published studies rather than miRNA array or bioinformatics method. More plasma miRNAs need to be analyzed to for using as specific biomarkers. Secondly, compare to single study, large sample and multicenter clinical trial studies will yield more reliable results. Moreover, there are still things for the further validation study need to be thought, including health policy, ethics, cost, et al.

\section{Conclusions}

In summary, this study suggests that the 10 plasma miRNAs are associated with lung cancer, and the changed expression levels could be used as potential biomarkers for diagnosis of lung cancer. SVM model has the superior diagnostic value for auxiliary diagnosis of lung cancer based on miRNAs tumor biomarkers and clinical symptom characteristics.

\section{Abbreviations}

Adenocarcinoma: AC; Artificial neural networks: ANN; Area under the ROC curve: AUC; Data mining: DM; decision tree: DT; Knowledge Discovery in Database: KDD; Large cell carcinoma: LCLC; Negative predictive value: NPV; Positron emission tomography: PET; Positive predictive value: PPV; Received operating characteristic: ROC; Small single-stranded non-coding RNAs: miRNAs; Support vector machine: SVM; Small cell lung cancer: SCLC; Non-small-cell lung cancer: NSCLC; Squamous cell carcinoma: SCC.

\section{Acknowledgements}

This study was supported by the Programs for National Nature Science Foundation of China (81001239, 81872597, 81473014), Science and Technology Development of Henan Province 
(142102310116) and the Outstanding Youth Grant of Zhengzhou University (1521329035).

\section{Ethical approval}

All procedures performed in studies involving human partcipants were in accordance to the ethical standards of the insttutonal and/or natonal research commitee and with the 1964 Helsinki Declaraton and its later amendments or comparable ethical standards.

\section{Competing Interests}

The authors have declared that no competing interest exists.

\section{References}

1. Miller KD, Siegel RL, Lin CC, Mariotto AB, Kramer JL, Rowland JH, et al. Cancer treatment and survivorship statistics, 2016. CA Cancer J Clin. 2016; 66: 271-89.

2. Zhu JF, Zhang $\mathrm{YL}$, Yang $\mathrm{X}$, Jin LT. Clinical Significance and Tumor-Suppressive Function of miR-516b in Nonsmall Cell Lung Cancer. Cancer Biother and Radiopharm. 2017; 32: 115-23.

3. Molina JR, Yang P, Cassivi SD, Schild SE, Adjei AA. Non-small cell lung cancer: epidemiology, risk factors, treatment, and survivorship. Mayo Clin Proc. 2008; 83: 584-94.

4. Foss KM, Sima C, Ugolini D, Neri M, Allen KE, Weiss GJ. miR-1254 and miR-574-5p: serum-based microRNA biomarkers for early-stage non-small cell lung cancer. J Thorac Oncol: official publication of the International Association for the Study of Lung Cancer. 2011; 6: 482-8.

5. Jemal A, Bray F, Center MM, Ferlay J, Ward E, Forman D. Global cancer statistics. CA Cancer J Clin. 2011; 61: 69-90.

6. Yang J, Zhu J, Zhang YH, Chen YS, Ding LL, Kensler TW, et al. Lung Cancer in a Rural Area of China: Rapid Rise in Incidence and Poor Improvement in Survival. Asian Pac J Cancer Prev. 2015; 16: 7295-302.

7. Swensen SJ, Jett JR, Hartman TE, Midthun DE, Mandrekar SJ, Hillman SL, et al. CT screening for lung cancer: five-year prospective experience. Radiology. 2005; 235: 259-65.

8. Aberle DR, Adams AM, Berg CD, Black WC, Clapp JD, et al. Reduced lung-cancer mortality with low-dose computed tomographic screening. N Engl J Med. 2011; 365: 395-409.

9. Cao JQ, Rodrigues GB, Louie AV, Zaric GS. Systematic review of the cost-effectiveness of positron-emission tomography in staging of non--small-cell lung cancer and management of solitary pulmonary nodules. Clin Lung Cancer. 2012; 13: 161-70.

10. Sharma S, Kelly TK, Jones PA. Epigenetics in cancer. Carcinogenesis. 2010; 31: 27-36

11. Yanaihara N, Harris CC. MicroRNA Involvement in Human Cancers. Clin Chem. 2013; 59: 1811-2

12. Switlik W, Karbownik MS, Suwalski M, Kozak J, Szemraj J. miR-30a-5p together with miR-210-3p as a promising biomarker for non-small cell lung cancer: A preliminary study. Cancer Biomarker. 2018; 21: 479-88.

13. Kunz M, Wolf B, Schulze H, Atlan D, Walles T, Walles H, et al. Non-Coding RNAs in Lung Cancer: Contribution of Bioinformatics Analysis to the Development of Non-Invasive Diagnostic Tools. Genes. 2016; 8: 8.

14. Geng Q, Fan T, Zhang B, Wang W, Xu Y, Hu H. Five microRNAs in plasma as novel biomarkers for screening of early-stage non-small cell lung cancer. Respir Res. 2014; 15: 149.

15. Inamura K, Ishikawa Y. MicroRNA In Lung Cancer: Novel Biomarkers and Potential Tools for Treatment. J Clin Med. 2016; 5: 36.

16. Zheng DL, Haddadin S, Wang Y, Gu LQ, Perry MC, Freter CE, et al. Plasma microRNAs as novel biomarkers for early detection of lung cancer. Int J Clin Exp Pathol. 2011; 4: 575-86.

17. Fang H, Lu B, Wang X, Zheng L, Sun K, Cai W. Application of data mining techniques to explore predictors of upper urinary tract damage in patients with neurogenic bladder. Braz J Med Biol Res. 2017; 50: e6638.

18. Chen HL, Yang B, Liu J, Liu DY. A support vector machine classifier with rough set-based feature selection for breast cancer diagnosis. Expert Syst Appl. 2011; 38: 9014-22.

19. Jayasurya K, Fung G, Yu S, Dehing-Oberije C, De Ruysscher D, Hope A, et al. Comparison of Bayesian network and support vector machine models for two-year survival prediction in lung cancer patients treated with radiotherapy. Med Phys. 2010; 37: 1401-7.

20. Wang W, Feng XL, Duan XR, Tan SJ, Wang SH, Wang TW, et al. Establishment of two data mining models of lung cancer screening based on three gene promoter methylations combined with telomere damage. Int J Biol Markers. 2017; 32: E141-E6.

21. Wang K, Yuan Y, Cho JH, McClarty S, Baxter D, Galas DJ. Comparing the MicroRNA Spectrum between Serum and Plasma. PloS one 2012: 7: e41561.
22. Zhou X, Wen $W$, Zhu J, Huang ZB, Zhang L, Zhang H, et al. A six-microRNA signature in plasma was identified as a potential biomarker in diagnosis of esophageal squamous cell carcinoma. Oncotarget. 2017; 8: 34468-80.

23. Shen J, Todd NW, Zhang H, Yu L, Lingxiao X, Mei YP, et al. Plasma microRNAs as potential biomarkers for non-small-cell lung cancer. Lab Invest. 2011; 91: 579-87.

24. Zhou B, Wang D, Sun G, Mei F, Cui Y, Xu H. Effect of miR-21 on Apoptosis in Lung Cancer Cell Through Inhibiting the PI3K/ Akt/NF-kappaB Signaling Pathway in Vitro and in Vivo. Cell Physiol Biochem. 2018; 46: 999-1008.

25. Zhao FY, Han J, Chen XW, Wang J, Wang XD, Sun JG, et al. miR-223 enhances the sensitivity of non-small cell lung cancer cells to erlotinib by targeting the insulin-like growth factor-1 receptor. Int J Mol Med. 2016; 38: 183-91.

26. Li B, Ding CM, Li YX, Peng JC, Geng N, Qin WW. MicroRNA145 inhibits migration and induces apoptosis in human nonsmall cell lung cancer cells through regulation of the EGFR/PI3K/AKT signaling pathway. Oncol Rep. 2018; 40: 2944-54.

27. Jia Z, Zhang Y, Xu Q, Guo W, Guo A. miR-126 suppresses epithelial-to-mesenchymal transition and metastasis by targeting PI3K/AKT/Snail signaling of lung cancer cells. Oncol Lett. 2018; 15: 7369-75.

28. Meng F, Wang F, Wang L, Wong SC, Cho WC, Chan LW. MiR-30a-5p Overexpression May Overcome EGFR-Inhibitor Resistance through Regulating PI3K/AKT Signaling Pathway in Non-small Cell Lung Cancer Cell Lines. Front Genet. 2016; 7: 197.

29. Fujita Y, Yagishita S, Hagiwara K, Yoshioka Y, Kosaka N, Takeshita F, et al. The clinical relevance of the miR-197/CKS1B/STAT3-mediated PD-L1 network in chemoresistant non-small-cell lung cancer. Mol Ther. 2015; 23: 717-27.

30. Zhang Z, Wang J, Cheng J, Yu X. Effects of miR-126 on the STAT3 signaling pathway and the regulation of malignant behavior in lung cancer cells. Oncol lett. 2018; 15: 8412-6.

31. Peng Z, Pan L, Niu Z, Li W, Dang X, Wan L, et al. Identification of microRNAs as potential biomarkers for lung adenocarcinoma using integrating genomics analysis. Oncotarget. 2017; 8: 64143-56.

32. Alhoshani A, Alrashdi A, Alhosaini K, Alanazi FE, Alajez NM, Altaf M, et al. Gold-containing compound BDG-I inhibits the growth of A549 lung cancer cells through the deregulation of miRNA expression. Saudi Pharm J. 2018; 26: 1035-43.

33. Attwooll C, Lazzerini Denchi E, Helin K. The E2F family: specific functions and overlapping interests. EMBO J. 2004; 23: 4709-16.

34. Babu KR, Muckenthaler MU. miR-20a regulates expression of the iron exporter ferroportin in lung cancer. J Mol Med (Berl). 2016; 94: 347-59.

35. Dou L, Han K, Xiao M, Lv F. miR-223-5p Suppresses Tumor Growth and Metastasis in Non-Small Cell Lung Cancer by Targeting E2F8. Oncol Res. 2019; 27: 261-8.

36. Deng $\mathrm{T}$, Yuan $\mathrm{YZ}$, Zhang $\mathrm{CN}$, Zhang $\mathrm{CY}$, Yao WY, Wang $\mathrm{C}$, et al. Identification of Circulating MiR-25 as a Potential Biomarker for Pancreatic Cancer Diagnosis. Cell Physiol Biochem. 2016; 39: 1716-22.

37. Zhao ZY, Liu JT, Wang CL, Wang $Y$, Jiang $Y$, Guo M. MicroRNA-25 regulates small cell lung cancer cell development and cell cycle through cyclin E2. Int J Clin Exp Pathol. 2014; 7: 7726-34.

38. Pfeffer SR, Yang CH, Pfeffer LM. The Role of miR-21 in Cancer. Drug Dev Res. 2015; 76: 270-7.

39. Xu TX, Zhao SZ, Dong M, Yu XR. Hypoxia responsive miR-210 promotes cell survival and autophagy of endometriotic cells in hypoxia. Eur Rev Med Pharmacol Sci. 2016; 20: 399-406.

40. Puissegur MP, Mazure NM, Bertero T, Pradelli L, Grosso S, Robbe-Sermesant $\mathrm{K}$, et al. miR-210 is overexpressed in late stages of lung cancer and mediates mitochondrial alterations associated with modulation of HIF-1 activity. Cell Death Differ. 2011; 18: 465-78.

41. Fiori ME, Barbini C, Haas TL, Marroncelli N, Patrizii M, Biffoni M, et al. Antitumor effect of miR-197 targeting in p53 wild-type lung cancer. Cell Death Differ. 2014; 21: 774-82.

42. Cho WC, Chow AS, Au JS. MiR-145 inhibits cell proliferation of human lung adenocarcinoma by targeting EGFR and NUDT1. RNA Biol. 2011; 8: 125-31.

43. Zhang H, Mao F, Shen TY, Luo QQ, Ding ZP, Qian LQ, et al. Plasma miR-145, miR-20a, miR-21 and miR-223 as novel biomarkers for screening early-stage non-small cell lung cancer. Oncol lett. 2017; 13: 669-76.

44. Miko E, Margitai Z, Czimmerer Z, Varkonyi I, Dezso B, Lanyi A, et al. miR-126 inhibits proliferation of small cell lung cancer cells by targeting SLC7A5. FEBS lett. 2011; 585: 1191-6.

45. Kumar R, Kumari B, Kumar M. Prediction of endoplasmic reticulum resident proteins using fragmented amino acid composition and support vector machine. PeerJ. 2017: 5: e3561.

46. Chen D, Guo W, Qiu Z, Wang Q, Li Y, Liang L, et al. MicroRNA-30d-5p inhibits tumour cell proliferation and motility by directly targeting CCNE2 in non-small cell lung cancer. Cancer Lett. 2015; 362: 208-17.

47. Amani M, Amani P, Kasaeian A, Mahian O, Pop I, Wongwises S. Modeling and optimization of thermal conductivity and viscosity of $\mathrm{MnFe} 2 \mathrm{O} 4$ nanofluid under magnetic field using an ANN. Sci Rep. 2017; 7: 17369

48. Hung CI, Lee PL, Wu YT, Chen LF, Yeh TC, Hsieh JC. Recognition of motor imagery electroencephalography using independent component analysis and machine classifiers. Ann Biomed Eng. 2005; 33: 1053-70.

49. Sattari MT, Farkhondeh A, Patrick Abraham J. Estimation of sodium adsorption ratio indicator using data mining methods: a case study in Urmia Lake basin, Iran. Environ Sci Pollut Res Int. 2018; 25: 4776-86. 
50. Guo L, Liu X, Wu Y, Yan W, Shen X. Research on the segmentation of MRI image based on multi-classification support vector machine. Conf Proc IEEE Eng Med Bio Soc. 2007; 2007: 6020-3.

51. Nedaie A, Najafi AA. Support vector machine with Dirichlet feature mapping. Neural Netw. 2017; 98: 87-101.

52. Shi GL, Chen Y, Sun Y, Yin YJ, Song CX. Significance of Serum MicroRNAs in the Auxiliary Diagnosis of Non-Small Cell Lung Cancer. Clin Lab. 2017; 63: $133-40$.

53. Wang Z, Feng F, Zhou X, Duan L, Wang J, Wu Y, et al. Development of diagnostic model of lung cancer based on multiple tumor markers and data mining. Oncotarget. 2017; 8: 94793-804.

54. Zhang H, Mao F, Shen T, Luo Q, Ding Z, Qian L, et al. Plasma miR-145, miR-20a, miR-21 and miR-223 as novel biomarkers for screening early-stage non-small cell lung cancer. Oncol lett. 2017; 13: 669-76.

55. Zhang $\mathrm{YH}$, Huang $\mathrm{T}$, Chen $\mathrm{L}, \mathrm{Xu} \mathrm{Y,} \mathrm{Hu} \mathrm{Y,} \mathrm{Hu} \mathrm{LD,} \mathrm{et} \mathrm{al.} \mathrm{Identifying} \mathrm{and}$ analyzing different cancer subtypes using RNA-seq data of blood platelets. Oncotarget. 2017; 8: 87494-511.

56. Li S, Zeng X, Ma R, Wang L. MicroRNA-21 promotes the proliferation, migration and invasion of non-small cell lung cancer A549 cells by regulating autophagy activity via AMPK/ULK1 signaling pathway. Exp Ther Med. 2018; 16: 2038-45.

57. Zhang H, Chen F, He Y, Yi L, Ge C, Shi X, et al. Sensitivity of non-small cell lung cancer to erlotinib is regulated by the Notch/miR-223/FBXW7 pathway. Biosci Rep. 2017; 37: BSR20160478.

58. Huang L, Li F, Deng P, Hu C. MicroRNA-223 Promotes Tumor Progression in Lung Cancer A549 Cells via Activation of the NF-kappaB Signaling Pathway. Oncol Res. 2016; 24: 405-13.

59. Han J, Zhao F, Zhang J, Zhu H, Ma H, Li X, et al. miR-223 reverses the resistance of EGFR-TKIs through IGF1R/PI3K/Akt signaling pathway. Int J Oncol. 2016; 48: 1855-67.

60. Ding $\mathrm{X}$, Zhong $\mathrm{T}$, Jiang $\mathrm{L}$, Huang $\mathrm{J}$, Xia $\mathrm{Y}, \mathrm{Hu}$ R. miR-25 enhances cell migration and invasion in non-small-cell lung cancer cells via ERK signaling pathway by inhibiting KLF4. Mol Med Rep. 2018; 17: 7005-16.

61. Zhao Z, Liu J, Wang C, Wang Y, Jiang Y, Guo M. MicroRNA-25 regulates small cell lung cancer cell development and cell cycle through cyclin E2. Int J Clin Exp Pathol. 2014; 7: 7726-34.

62. Yu W, Ding J, He M, Chen Y, Wang R, Han Z, et al. Estrogen receptor beta promotes the vasculogenic mimicry $(\mathrm{VM})$ and cell invasion via altering the IncRNA-MALAT1/miR-145-5p/NEDD9 signals in lung cancer. Oncogene. 2019; 38: 1225-38.

63. Chang Y, Yan W, Sun C, Liu Q, Wang J, Wang M. miR-145-5p inhibits epithelial-mesenchymal transition via the JNK signaling pathway by targeting MAP3K1 in non-small cell lung cancer cells. Oncol lett. 2017; 14: 6923-8.

64. Li JC, Zheng JQ. Effect of microRNA-145 on proliferation and apoptosis of human non-small cell lung cancer A549 cells by regulating mTOR signaling pathway. J Cell Biochem. 2017.DOI: 10.1002/jcb.26629. 